\title{
THE BLOCH-KATO CONJECTURE FOR ADJOINT MOTIVES OF MODULAR FORMS
}

\author{
Fred Diamond, Matthias Flach, and Li Guo
}

\begin{abstract}
The Tamagawa number conjecture of Bloch and Kato describes the behavior at integers of the $L$-function associated to a motive over $\mathbf{Q}$. Let $f$ be a newform of weight $k \geq 2$, level $N$ with coefficients in a number field $K$. Let $M$ be the motive associated to $f$ and let $A$ be the adjoint motive of $M$. Let $\lambda$ be a finite prime of $K$. We verify the $\lambda$-part of the Bloch-Kato conjecture for $L(A, 0)$ and $L(A, 1)$ when $\lambda \nmid N k$ ! and the mod $\lambda$ representation associated to $f$ is absolutely irreducible when restricted to the Galois group over $\mathbf{Q}\left(\sqrt{(-1)^{(\ell-1) / 2} \ell}\right)$ where $\lambda \mid \ell$.
\end{abstract}

\section{Introduction}

This is a summary of results on the Tamagawa number conjecture of Bloch and Kato [B-K] for adjoint motives of modular forms of weight $k \geq 2$. The conjecture relates the value at 0 of the associated $L$-function to arithmetic invariants of the motive. We prove in [D-F-G] that it holds up to powers of certain "bad primes." The strategy for achieving this is essentially due to Wiles [Wi], as completed with Taylor in $[\mathrm{T}-\mathrm{W}]$. The Taylor-Wiles construction yields a formula relating the size of a certain module measuring congruences between modular forms to that of a certain Galois cohomology group. This was carried out in [Wi] and $[\mathrm{T}-\mathrm{W}]$ in the context of modular forms of weight 2, where it was used to prove results in the direction of the Fontaine-Mazur conjecture [F-M]. While it was no surprise that the method could be generalized to higher weight modular forms and that the resulting formula would be related to the Bloch-Kato conjecture, there remained many technical details to verify in order to accomplish this. In particular, the very formulation of the conjecture relies on a comparison isomorphism between the $\ell$-adic and de Rham realizations of the motive provided by theorems of Faltings $[\mathrm{Fa}]$ or Tsuji $[\mathrm{Ts}]$, and verification of the conjecture requires the careful application of such a theorem. We also need to generalize results on congruences between modular forms to higher weight, and to compute certain local Tamagawa numbers.

Received March 24, 2001.

1991 Mathematics Subject Classification. Primary 11F67, 11F80, 11G40; Secondary $14 \mathrm{G} 10,14 \mathrm{~F}, 19 \mathrm{~F} 27$.

Key words and phrases. modular forms, Bloch-Kato conjecture, adjoint motive. 


\section{The adjoint motive}

Suppose $f$ is a newform of weight $k \geq 2$, level $N$ and character $\psi$ with coefficients in the ring of integers $\mathcal{O}$ of a number field $K$. For $*=B$, dr or $\lambda$ (a prime of $K$ ), we let $M_{*}$ denote the corresponding realization of the motive attached to $f$ (see [De2]). This is a two-dimensional $K_{*}$-subspace (where $K_{B}=K_{\mathrm{dr}}=K$ ) of the corresponding cohomology group of the modular curve $X_{1}(N)$ with coefficients in a sheaf depending on $k$. We have certain additional structure on each $M_{*}$ (an involution, filtration or $G_{\mathbf{Q}^{-}}$-action) and comparison isomorphisms relating certain pairs of realizations. Let $S$ be a set of primes containing those dividing $N k$ !, and let $\mathcal{O}_{B}=\mathcal{O}_{\mathrm{dr}}=\mathcal{O}_{S}$ denote the $S$-integers in $K$. For $*=B$, dr or $\lambda$ for $\lambda \notin S$, we also have natural $\mathcal{O}_{*}$-lattices $\mathcal{M}_{*}$ in $M_{*}$ and integral versions of the comparison isomorphisms involving the $\mathcal{M}_{\lambda}$.

Let $D_{*}$ denote the $*$-realization of the $(1-k)$-Tate twist of the motive associated to $\psi$. Poincaré duality for $X_{1}(N)$ gives rise to a perfect alternating pairing on $M_{*}$ with values in $D_{*}$, i.e., an isomorphism

$$
\mu_{*}: \wedge_{K_{*}}^{2} M_{*} \rightarrow D_{*}
$$

respecting the various additional structures and comparison isomorphisms. We also have natural $\mathcal{O}_{*}$-lattices $\mathcal{D}_{*}$ in $D_{*}$, but the map $\wedge_{\mathcal{O}_{*}}^{2} \mathcal{M}_{*} \rightarrow \mathcal{D}_{*}$ need not be an isomorphism; the image is $\eta \mathcal{D}_{*}$ for a certain ideal $\eta$ of $\mathcal{O}_{S}$. More generally, if $\Sigma$ is a finite set of primes contained in $S$, we analogously define $\mathcal{M}_{*}^{\Sigma}, \mu_{*}^{\Sigma}$ and $\eta^{\Sigma}$ using level structure which is, roughly speaking, minimal outside $\Sigma$.

We let $A_{*}$ denote the set of trace-zero elements in $\operatorname{End}_{K_{*}} M_{*}$. So $A_{*}$ is threedimensional over $K_{*}$ and we have comparison isomorphisms, including

$$
I_{\infty}: A_{\mathrm{dr}} \otimes \mathbf{C} \rightarrow A_{B} \otimes \mathbf{C},
$$

induced by those for the $M_{*}$. Replacing $f$ by a twist does not change this data, so we always assume $f$ has minimal conductor among its twists. The $L$-function associated to $A_{\lambda}$ is independent of $\lambda$; we denote it $L(A, s)$ and regard it as taking values in $K \otimes \mathbf{C}$. We know also that $L(A, s)$ extends to an entire function on the complex plane and satisfies the functional equation (see [G-J], [Sc]),

$$
\Lambda(A, s)=\epsilon(A, s) \Lambda(A, 1-s),
$$

where

$$
\Lambda(A, s)=L(A, s) \Gamma_{\mathbf{R}}(s+1) \Gamma_{\mathbf{C}}(s+k-1)
$$

and $\epsilon(A, s)$ is as defined by Deligne [De1]. Moreover $L(A, 0)$ and $L(A, 1)$ are invertible elements of $\mathbf{R} \otimes K$, and $\epsilon(A, s)= \pm C^{1-2 s}$ for some positive integer $C$ dividing $N$.

\section{The Bloch-Kato conjecture}

We show that the conjecture of Bloch and Kato $[\mathrm{B}-\mathrm{K}]$ correctly predicts the values of $L(A, 0)$ and $L(A, 1)$ up to an element of $\mathcal{O}_{S}^{\times}$, where $S$ is a certain finite set of primes containing those dividing $N k$ !. We use the formulation of the 
conjecture given by Fontaine and Perrin-Riou [F-P], which is easily generalized to the setting of $K$-coefficients.

The fundamental line for $A$ is the $K$-line defined by

$$
\begin{aligned}
\Delta & =\operatorname{Hom}_{K}\left(A_{B}^{+}, A_{\mathrm{dr}} / \mathrm{Fil}^{0} A_{\mathrm{dr}}\right) \\
& \cong \operatorname{Hom}_{K}\left(\mathrm{Fil}^{k-1} M_{\mathrm{dr}}, M_{\mathrm{dr}} / \mathrm{Fil}^{k-1} M_{\mathrm{dr}}\right) .
\end{aligned}
$$

The Deligne period for $A$ is the natural basis $c^{+}$for $\Delta \otimes \mathbf{R}$ gotten from $I_{\infty}$. One finds that $c^{+} L(A, 0)^{-1}$ is an element $\delta \in \Delta$ characterized by

$$
\mu_{\mathrm{dr}}(f \wedge \delta(f))=\frac{i^{k-\eta}((k-2) !)^{2} \epsilon(M) \prod_{p \in P}\left(1+p^{-1}\right)}{2 \epsilon(D) \epsilon(A)} \cdot G_{\mathrm{dr}},
$$

where $\eta \in\{0,1\}$ has the same parity as $k, P$ is a certain exceptional set of primes dividing $N$ and $G_{\mathrm{dr}}$ (for Gauss sum) is the usual basis for $D_{\mathrm{dr}}$. This is proved by relating the Deligne period to the Petersson product, applying a method of Rankin and Shimura to relate this to $L(A, 1)$ (see [St], [Hi], [Sc]) and using the functional equation.

To state the $\lambda$-part of the Bloch-Kato conjecture, one chooses a Galois-stable $\mathcal{O}_{\lambda}$-lattice $\mathcal{A}_{\lambda}$ in $A_{\lambda} \cong A_{B} \otimes_{K} K_{\lambda}$ and an $\mathcal{O}_{\lambda}$-lattice $\omega$ in $\left(A_{\mathrm{dr}} / \mathrm{Fil}^{0} A_{\mathrm{dr}}\right) \otimes_{K} K_{\lambda}$. The conjecture, which turns out to be independent of these choices, states that

- $H_{f}^{1}\left(G_{\mathbf{Q}}, A_{\lambda} / \mathcal{A}_{\lambda}\right)$ is finite;

- $\delta$ generates the $\mathcal{O}_{\lambda}$-submodule

$$
\frac{\operatorname{Fitt}_{\mathcal{O}_{\lambda}} H^{0}\left(G_{\mathbf{Q}}, A_{\lambda} / \mathcal{A}_{\lambda}\right) \cdot \operatorname{Fitt}_{\mathcal{O}_{\lambda}} H^{0}\left(G_{\mathbf{Q}}, B_{\lambda} / \mathcal{B}_{\lambda}\right)}{\operatorname{Tam}_{\omega}^{0}\left(\mathcal{A}_{\lambda}\right) \cdot \operatorname{Fitt}_{\mathcal{O}_{\lambda}} H_{f}^{1}\left(G_{\mathbf{Q}}, A_{\lambda} / \mathcal{A}_{\lambda}\right)} \cdot \operatorname{Hom}_{\mathcal{O}_{\lambda}}\left(\mathcal{A}_{\lambda}^{+}, \omega\right)
$$

of $\Delta \otimes_{K} K_{\lambda}$, where $\mathcal{B}_{\lambda}=\operatorname{Hom}_{\mathbf{Z}_{\ell}}\left(\mathcal{A}_{\lambda}, \mathbf{Z}_{\ell}(1)\right), B_{\lambda}=\mathcal{B}_{\lambda} \otimes \mathbf{Q}$ and $\operatorname{Tam}_{\omega}^{0}\left(\mathcal{A}_{\lambda}\right)$ is the Tamagawa ideal of $\mathcal{A}_{\lambda}$ relative to $\omega$.

\section{Methods}

The two main problems are to compute the local contribution at $\ell$ to the Tamagawa ideal, $\operatorname{Tam}_{\ell, \omega}\left(\mathcal{A}_{\lambda}\right)$, and the length of $H_{f}^{1}\left(G_{\mathbf{Q}}, A_{\lambda} / \mathcal{A}_{\lambda}\right)$. We restrict our attention to primes $\lambda$ not in $S$, and let $\mathcal{A}_{*}$ denote the set of elements of $\operatorname{End}_{\mathcal{O}_{*}}\left(\mathcal{M}_{*}\right)$ of trace zero. One can then use existing machinery to show that $\operatorname{Tam}_{\ell, \omega}\left(\mathcal{A}_{\lambda}\right)=\mathcal{O}_{\lambda}$, where $\omega=\left(\mathcal{A}_{\mathrm{dr}} / \mathrm{Fil}^{0} \mathcal{A}_{\mathrm{dr}}\right) \otimes_{\mathcal{O}_{\mathrm{dr}}} \mathcal{O}_{\lambda}$. The proof requires only that $\lambda$ not divide $N k$ ! and uses the integral version of Faltings' comparison theorem [Fa]. For primes between $k$ and $2 k$, the argument is slightly delicate since the filtration length of $A_{\mathrm{dr}}$ is greater than $\ell-2$.

The computation of the $H_{f}^{1}$ relies on the methods of Wiles [Wi] and TaylorWiles [T-W], as modified in [Di1] and [Di2]. Now we have to impose another hypothesis on $\lambda$ :

(2) $\bar{\rho}: G_{\mathbf{Q}} \rightarrow \operatorname{Aut}_{\mathcal{O}_{\lambda} / \lambda}\left(\mathcal{M}_{\lambda} / \lambda \mathcal{M}_{\lambda}\right)$ has absolutely irreducible restriction to $G_{L}$, where $L=\mathbf{Q}\left(\sqrt{(-1)^{(\ell-1) / 2} \ell}\right)$. 
We give an axiomatic formulation of the method of the following nature: Suppose

$$
\mathcal{R}=\left\{\rho: G_{\mathbf{Q}} \rightarrow \operatorname{Aut}_{K_{\rho}} V_{\rho}\right\}
$$

is a set of liftings of $\bar{\rho}$, where for each $\rho$ in $\mathcal{R}, K_{\rho}$ is a finite extension of $K_{\lambda}$ in $\bar{K}_{\lambda}$ and the restriction of $\rho$ to $G_{\mathbf{Q}_{\ell}}$ is crystalline of Hodge-Tate type $(0, k-1)$ (see $[\mathrm{Fo}]$ ). For any finite set of primes $\Sigma$ not containing $\ell$, let $\mathcal{R}^{\Sigma}$ denote the set of $\rho \in \mathcal{R}$ such that $\rho$ is minimally ramified outside $\Sigma$. Suppose that for each $\rho \in \mathcal{R}^{\Sigma}$ we are given an isomorphism

$$
\mu_{\rho}^{\Sigma}: \wedge_{K_{\rho}}^{2} V_{\rho} \rightarrow D_{\lambda} \otimes_{K_{\lambda}} K_{\rho}
$$

We assume that for fixed $\rho$ and varying $\Sigma$, the ratios of the $\mu_{\rho}^{\Sigma}$ are determined by Euler factors. We assume also that $\oplus_{\rho \in \mathcal{R}}\left(V_{\rho} \otimes_{K_{\rho}} \bar{K}_{\lambda}\right)$ contains a lattice $\mathcal{V}$ having a certain self-duality property with respect to the pairings. We show that if the numbers $\# \mathcal{R}^{\Sigma}$ satisfy a certain numerical criterion, then

- every lifting of $\bar{\rho}$ which is crystalline of Hodge-Tate type $(0, k-1)$ is isomorphic to an element of $\mathcal{R}$;

- if $\rho \in \mathcal{R}^{\Sigma}$ and $K_{\rho}=K_{\lambda}$, then

$$
\operatorname{Fitt}_{\mathcal{O}_{\lambda}} H_{\Sigma}^{1}\left(G_{\mathbf{Q}}, \mathcal{W}_{\rho} \otimes_{\mathcal{O}_{\lambda}}\left(K_{\lambda} / \mathcal{O}_{\lambda}\right)\right)=\operatorname{Fitt}_{\mathcal{O}_{\lambda}} \mathcal{D}_{\lambda} / \mu_{\rho}^{\Sigma}\left(\wedge_{\mathcal{O}_{\lambda}}^{2} \mathcal{V}_{\rho}\right)
$$

where $\mathcal{V}_{\rho}=\mathcal{V} \cap V_{\rho}$ and $\mathcal{W}_{\rho}$ is the set of elements of $\operatorname{End}_{\mathcal{O}_{\lambda}} \mathcal{V}_{\rho}$ of trace zero.

\section{Main results}

Generalizing results and methods of Ribet, Wiles and others ([Ri], [D-T], [Wi], [T-W], [Di1]), we verify that these hypotheses are satisfied by the set $\mathcal{R}$ of liftings of $\bar{\rho}$ associated to newforms of weight $k$, level prime to $\ell$ and character $\psi$. We thus obtain the following result in the direction of the Fontaine-Mazur conjecture $[\mathrm{F}-\mathrm{M}]$ :

Theorem 1. Suppose $\rho: G_{\mathbf{Q}} \rightarrow$ Aut $_{K_{\lambda}} V_{\lambda}$ is continuous, two-dimensional, unramified outside a finite set of primes and has crystalline restriction to $G_{\mathbf{Q}_{\ell}}$ of Hodge-Tate type $(0, d)$ with $0<d<\ell-1$. If $\bar{\rho}$ is modular and has absolutely irreducible restriction to $G_{L}$, then $\rho$ is modular.

We also find that $\operatorname{Fitt}_{\mathcal{O}_{\lambda}} H_{\Sigma}^{1}\left(G_{\mathbf{Q}}, A_{\lambda} / \mathcal{A}_{\lambda}\right)=\eta^{\Sigma} \mathcal{O}_{\lambda}$. Using the modified PoitouTate sequence (see [Fl2]), we can then compute the length of $H_{f}^{1}\left(G_{\mathbf{Q}}, A_{\lambda} / \mathcal{A}_{\lambda}\right)$, which is dual to $H_{f}^{1}\left(G_{\mathbf{Q}}, B_{\lambda} / \mathcal{B}_{\lambda}\right)$ [Fl1]. Note that (2) implies that $H^{0}\left(G_{\mathbf{Q}}, A_{\lambda} / \mathcal{A}_{\lambda}\right)$ and $H^{0}\left(G_{\mathbf{Q}}, B_{\lambda} / \mathcal{B}_{\lambda}\right)$ both vanish. Applying equation (1) and its analogue for $L(A, 1)$, we conclude:

Theorem 2. Let $f$ be a newform of weight $k \geq 2$ and level $N$ with coefficients in a number field $K$. Let $S$ denote the set of primes $\lambda$ such that $\lambda$ divides $N k$ ! or (2) fails. For $\lambda$ not in $S$, the $\lambda$-part of the Bloch-Kato conjecture holds for $L(A, 0)$ and $L(A, 1)$. 
Finally we remark that the set $S$ is finite. In fact, if $\lambda$ does not divide $N k$ ! and (2) fails, then $\ell=2 k-1, \ell=2 k-3$ or $\mathcal{M}_{\lambda} / \lambda \mathcal{M}_{\lambda}$ defines a reducible representation of $G_{\mathbf{Q}}$, and reducibility for infinitely many $\lambda$ would violate the Ramanujan conjecture.

\section{References}

[B-K] S. Bloch and K. Kato, L-functions and Tamagawa numbers of motives, The Grothendieck Festschrift, Vol. 1, Birkhäuser, 1990, pp. 333-400.

[De1] P. Deligne, Les constants des équations fonctionelles des fonctions L, Modular Functions of One Variable II, Lecture Notes in Math. 349, Springer-Verlag, 1973, pp. 501-595.

[De2] - Valeurs de fonctions L et périodes d'intégrales, Automorphic Forms, Representations and $L$-functions, Proc. Symp. Pure Math. 33, Amer. Math. Soc., 1979, pp. 313-346.

[Di1] F. Diamond, On deformation rings and Hecke rings, Ann. of Math. 144 (1996), $137-166$.

[Di2] $\quad$ The Taylor-Wiles construction and multiplicity one, Invent. Math. 128 (1997), 379-391.

[D-F-G] F. Diamond, M. Flach, and L. Guo, Adjoint motives of modular forms and the Tamagawa number conjecture, preprint.

[D-T] F. Diamond and R. Taylor, Lifting modular mod $\ell$ representations, Duke Math. J. 74 (1994), 253-269.

[Fa] G. Faltings, Crystalline cohomology and p-adic étale cohomology, Algebraic Analysis, Geometry and Number Theory, John Hopkins Univ. Press, Baltimore MD, 1989, pp. 25-80.

[F11] M. Flach, A generalization of the Cassels-Tate pairing, J. Reine Angew. Math. 412 (1990), 113-127.

[Fl2] Selmer groups for the symmetric square of an elliptic curve, Ph.D. dissertation, University of Cambridge, 1991.

[Fo] J.-M. Fontaine, Représentations p-adiques semi-stables, Périodes p-adiques, Astérisque 223 (1994), 113-184.

[F-M] J.-M. Fontaine and B. Mazur, Geometric Galois representations, Elliptic curves, modular forms and Fermat's last theorem, Internat. Press, 1995, pp. 41-78.

[F-P] J.-M. Fontaine and B. Perrin-Riou, Autour des conjectures de Bloch et Kato: cohomologie galoisienne et valeurs de fonction L, Motives, Proc. Sympos. Pure Math. 55, Part 1, Amer. Math. Soc., 1994, pp. 599-706.

[G-J] S. Gelbart and H. Jacquet, A relation between automorphic representations of GL(2) and GL(3), Ann. Sci. Ecole Norm. Sup 11 (1978), 471-542.

[Hi] H. Hida, Congruences of cusp forms and special values of their zeta functions, Invent. Math. 63 (1981), 225-261.

[Ri] K. Ribet, On modular representations of $\mathrm{Gal}(\overline{\mathbf{Q}} / \mathbf{Q})$ arising from modular forms, Invent. Math. 100 (1990), 431-476.

[Sc] C.-G. Schmidt, p-adic measures attached to automorphic representations of GL(3), Invent. Math. 92 (1988), 597-631.

[St] J. Sturm, Special values of zeta functions and Eisenstein series of half integral weight, Amer. J. Math. 102 (1980), 219-240.

[T-W] R. Taylor and A. Wiles, Ring theoretic properties of certain Hecke algebras, Ann. of Math. 141 (1995), 553-572.

[Ts] T. Tsuji, p-adic etale cohomology and crystalline cohomology in the semi-stable reduction case, Invent. Math. 137 (1999), 233-411. 
[Wi] A. Wiles, Modular elliptic curves and Fermat's Last Theorem, Ann. of Math. 141 (1995), 443-551.

Dept. of Mathematics, Brandeis University, Waltham, MA 02454.

E-mail address: fdiamond@brandeis.edu

Dept. of Mathematics, California Institute of Technology, Pasadena, CA 91125.

E-mail address: flachøcco.caltech.edu

Dept. of Mathematics and Computer Science, University of Rutgers at Newark, NEWARK, NJ 07102.

E-mail address: liguo@newark.rutgers.edu 